Mathematical Research Letters 1, 87-94 (1994)

\title{
DERIVED CATEGORIES AND MOTIVES
}

\author{
I. KRIZ AND J. P. MAY
}

\begin{abstract}
A в S т RA с т. We relate derived categories of modules over rational DGA's to categories of comodules over associated Hopf algebras, and we explain how this implies the equivalence of definitions of mixed Tate motives proposed by Bloch and Deligne. We also describe an approach to integral mixed Tate motives in terms of the derived categories of modules over certain $E_{\infty}$ algebras.
\end{abstract}

We first explain some new differential homological algebra - alias rational homotopy theory - over a field of characteristic zero and then use it to show the equivalence of two proposed definitions of mixed Tate motives $[8$, $9,5]$ in algebraic geometry. One of these has been proven to admit Hodge and étale realizations, but is intrinsically restricted to the rational world. The other can be linked up to a speculative definition of integral or modular mixed Tate motives, and we end by explaining some new differential homological algebra over arbitrary commutative rings that is necessary to make sense of this approach to mixed Tate motives.

Let $A$ be a commutative differential graded and "Adams graded" $k$ algebra, abbreviated DGA, where $k$ is a field of characteristic zero. Thus $A$ is bigraded via $k$-modules $A^{q}(r)$, where $q \in \mathbb{Z}$ and $r \geq 0$. We assume that $A^{q}(r)=0$ unless $2 r \geq q$. The differential and product behave as follows with respect to the gradings:

$$
d: A^{q}(r) \rightarrow A^{q+1}(r) \text { and } A^{q}(r) \otimes A^{s}(t) \rightarrow A^{q+s}(r+t) .
$$

We assume that $A$ has an augmentation $\varepsilon: A \rightarrow k$. Write $H^{q}(A)(r)$ for the cohomology of $A$ in bidegree $(q, r)$. We also assume throughout that $A$ is cohomologically connected, in the sense that $H^{q}(A)(r)=0$ if $q<0$,

1991 Mathematics Subject Classification. Primary: 14C25, 14C35, 18E30, 18G15, $19 \mathrm{E} 15$.

Received October 13, 1993.

The first author is an Alfred P. Sloan Fellow; both authors acknowledge support from the NSF. 
$H^{0}(A)(r)=0$ if $r>0$, and $\varepsilon$ induces an isomorphism $H^{0}(A)(0) \rightarrow k$. We say that $A$ is connected if $A^{q}=0$ for $q<0$ and $A^{0} \cong k$. Any cohomologically connected DGA is quasi-isomorphic to a connected DGA. While the Adams grading is present and important in our motivating examples, our homological results apply verbatim to DGA's without Adams grading.

Let $\mathscr{D}_{A}$ denote the derived category of cohomologically bounded below $A$-modules. Its objects are differential bigraded $A$-modules $M$, where $M^{q}(r)$ may be non-zero for any pair of integers $(q, r)$, such that $H^{q}(M)(r)=$ 0 for all sufficiently small $q$. All of our $A$-modules are assumed to satisfy this cohomological condition. The morphisms of $\mathscr{D}_{A}$ are obtained by adjoining formal inverses to the quasi-isomorphisms in the homotopy category of maps of differential $A$-modules. Write $\otimes_{A}$ for the derived tensor product in $\mathscr{D}_{A}$. It is obtained by first applying a suitable projective (or cell - see below) approximation to modules and then applying the ordinary tensor product. Define the "indecomposable elements $Q M$ " by setting $Q M=k \otimes_{A} M$. Then $Q M$ is a differential bigraded $k$-module. Define $\mathscr{H}_{A}$ to be the full subcategory of $\mathscr{D}_{A}$ consisting of those $M$ such that $H^{q}(Q M)=0$ for $q \neq 0$. Define $\mathscr{F} \mathscr{H}_{A}$ to be the full subcategory of $\mathscr{H}_{A}$ consisting of the modules $M$ such that $H^{0}(Q M)$ is finite dimensional and define $\omega(M)=H^{0}(Q M)$. Recall the notion of a $t$-structure and its heart from $[1, \S 1.3]$ and the notion of a neutral Tannakian category from [11, $2.19]$ or [7].

Theorem 1. The triangulated category $\mathscr{D}_{A}$ admits a t-structure whose heart is $\mathscr{H}_{A}$, and $\mathscr{F}_{A} \mathscr{H}_{A}$ is a (graded) neutral Tannakian category over $k$ with fiber functor $\omega$.

In the special case when $A$ is a polynomial algebra on finitely many generators of even positive degree, most of this is proven in [2, pp. 93-103]. It follows from $[11,2.11]$ that the Abelian category $\mathscr{F}_{\mathcal{H}}$ is equivalent (in possibly many ways) to the category of finite dimensional representations of an affine group scheme. What amounts to the same thing $[11$, 2.2], $\mathscr{F} \mathscr{H}_{A}$ is equivalent to the category of finite dimensional comodules over a Hopf algebra (or bialgebra). We can specify such a Hopf algebra explicitly, without recourse to Tannakian theory. The algebra $A$ has a bar construction $\bar{B}(A)$. If $I A$ is the augmentation ideal of $A$, then $\bar{B}^{q}(A)(r)$ is the direct sum over $p \geq 0$ of the submodules of the $p$-fold tensor power of $I A$ in bigrading $(q+p, r)$. If $A$ is connected, $\bar{B}^{0}(A)$ is additively isomorphic to the tensor algebra on the (Adams graded) $k$-module $A^{1}$. We define $\chi_{A}=H^{0} \bar{B}(A)$. This is a commutative Hopf algebra, and it turns out to be a polynomial algebra. Its $k$-module of indecomposable elements is a co-Lie algebra, which we denote by $\gamma_{A}$. We think of $\chi_{A}$ as a kind of universal 
enveloping Hopf algebra of $\gamma_{A}$. The following theorem gives a concrete and explicit description of the category $\mathscr{H}_{A}$.

Theorem 2. Let $A$ be a connected DGA. Then the following Abelian categories are equivalent.

(1) The heart $\mathscr{H}_{A}$ of $\mathscr{D}_{A}$.

(2) The category of generalized nilpotent representations of the co-Lie algebra $\gamma_{A}$.

(3) The category of comodules over the Hopf algebra $\chi_{A}$.

(4) The category $\mathscr{T}_{A}$ of "generalized nilpotent twisting matrices in A".

We shall not make the definition of $\mathscr{T}_{A}$ explicit, but the relevant matrices are quite concrete combinatorial objects. The hypothesis that $A$ be connected and not just cohomologically connected is needed to allow use of $\mathscr{T}_{A}$. The other three categories are invariant under quasi-isomorphisms of cohomologically connected DGA's. The DGA $A$ has a "1-minimal model" $\iota: A\langle 1\rangle \rightarrow A$. The map $\iota$ induces an isomorphism on $H^{1}$ and a monomorphism on $H^{2}$. A quick construction is to let $A\langle 1\rangle$ be the exterior algebra on a copy of $\gamma_{A}$ concentrated in (homological) degree one, with differential induced by the cobracket on $\gamma_{A}$. We say that " $A$ is a $K(\pi, 1)$ " if $\iota$ is a quasi-isomorphism. The equivalence of (1) and (2) in Theorem 2 makes it clear that $\mathscr{H}_{A}$ depends only on $A\langle 1\rangle$.

Theorem 3. The derived category of bounded below chain complexes in $\mathscr{H}_{A}$ is equivalent to the derived category $\mathscr{D}_{A\langle 1\rangle}$.

Let $k(r)$ be a copy of $k$ concentrated in bidegree $(0, r)$, regarded as a representation of $\gamma_{A}$ in the evident way.

Corollary 4. If $A$ is a $K(\pi, 1)$, then

$$
\operatorname{Ext}_{\mathscr{H}_{A}}^{q}(k, k(r)) \cong H^{q}(A)(r) .
$$

While minimal algebras were introduced by Sullivan [15] in 1978 and have played a central role in rational homotopy theory ever since, the concomitant theory of minimal modules does not seem to appear in the literature and plays an important role in the proofs of the results above. Another basic ingredient, not special to characteristic zero, is a new general approach to derived categories that mimics the way topologists think about the stable homotopy category of spectra. There is a theory of "cell modules" that is just like the theory of "cell spectra", and there are simple algebraic analogues of such standard topological results as the Whitehead theorem and Brown's representability theorem. Derived tensor products of modules work in exactly the same way as smash products of spectra.

We do not believe that these results are best possible. We believe that the following result is true, and we have a sketch proof. 
Conjecture 5. There is an Abelian category $\mathscr{R}_{A}$ and an embedding $\iota$ of $\mathscr{D}_{A}$ as a full subcategory of the derived category of bounded below chain complexes in $\mathscr{R}_{A}$ such that $\iota(A(r))$ is an object of $\mathscr{R}_{A}$ and

$$
\operatorname{Ext}_{\mathscr{R}_{A}}^{q}(\iota(A), \iota(A(r))) \cong H^{q}(A)(r) .
$$

While the results above are statements in differential homological algebra, we formulated them as general results that would have to be true if two seemingly different definitions of mixed Tate motives were to agree. We briefly explain the relevance to mixed Tate motives. Let $X$ be a (smooth, quasi-projective) variety over a field $F$. Bloch [3] defined an Adams graded simplicial Abelian group $\mathscr{Z}(X)$ whose homology groups are the Chow groups of $X$ :

$$
C H^{r}(X, q)=H_{q}(\mathscr{Z}(X))(r) .
$$

Bloch $[3,4]$ (see also Levine [13]) proved that

$$
C H^{r}(X, q) \otimes \mathbb{Q} \cong\left(K_{q}(X) \otimes \mathbb{Q}\right)^{(r)},
$$

where the right side is the $n^{r}$-eigenspace of the Adams operation $\psi^{n}$ (for any $n>1) ; K_{q}(X) \otimes \mathbb{Q}$ is the direct sum of these eigenspaces.

The simplicial Abelian group $\mathscr{Z}(X)$ has a partially defined intersection product. At this point another aspect of our work enters. Specializing a general theory of "partial algebras", we have constructed an " $E_{\infty}$ algebra" $\mathscr{A}(X)$ that is quasi-isomorphic to the associated chain complex of $\mathscr{Z}(X)$. Here $E_{\infty}$ algebras are defined in terms of actions by operads of chain complexes and are the precise algebraic analogues of $E_{\infty}$ ring spectra in topology [14], [12]. We have also constructed a commutative rational DGA $\mathscr{A}_{\mathbb{Q}}(X)$ and a quasi-isomorphism of $E_{\infty}$ algebras

$$
\mathscr{A}(X) \otimes \mathbb{Q} \rightarrow \mathscr{A}_{\mathbb{Q}}(X) .
$$

These objects are graded homologically. Cohomological considerations dictate the regrading

$$
\mathscr{N}^{2 r-p}(X)(r)=\mathscr{A}_{p}(X)(r) \text { and } \mathscr{N}_{\mathbb{Q}}^{2 r-p}(X)(r)=\left(\mathscr{A}_{\mathbb{Q}}\right)_{p}(X)(r) .
$$

Since $\mathscr{A}_{p}(X)=0$ if $p<0, \mathscr{A}^{q}(X)(r)=0$ unless $2 r \geq q$. Thinking of the eigenspaces on the right side of (7) as successive terms of the associated graded with respect to the $\gamma$-filtration, we may rewrite $(7)$ in the form

$$
H^{q}\left(\mathscr{N}_{\mathbb{Q}}(X)\right)(r)=g r_{\gamma}^{r}\left(K_{2 r-q}(X) \otimes \mathbb{Q}\right) .
$$


The Beilinson-Soulé conjecture for $X$ asserts that these groups are zero if $q<0$ or if $q=0$ and $r \neq 0$, and that the group in bidegree $(0,0)$ is $\mathbb{Q}$. That is, the Beilinson-Soulé conjecture is that $\mathscr{N}_{\mathbb{Q}}(X)$ is cohomologically connected. It holds when $F$ is a number field [6]. When it holds, our results above apply to $A=\mathscr{N}_{\mathbb{Q}}(X)$.

Specializing to $X=\operatorname{Spec}(F)$, let $\mathscr{N}$ denote the $E_{\infty}$ algebra $\left.\mathscr{N} \operatorname{Spec}(F)\right)$ and let $\mathscr{N}_{\mathbb{Q}}$ denote the commutative DGA $\mathscr{N}_{\mathbb{Q}}(\operatorname{Spec}(F))$. Even without the Beilinson-Soulé conjecture, [5] proposed the following definition.

Definition 10. Let $\chi_{m o t}$ denote the Hopf algebra $\chi_{N_{\mathbb{Q}}}=H^{0} \bar{B}\left(\mathscr{N}_{\mathbb{Q}}\right)$. Define the category of (rational) mixed Tate motives of the field $F$, denoted $\mathscr{M} \mathscr{T} \mathscr{M}(F)$, to be the category of finite dimensional comodules over $\chi_{\text {mot }}$.

Such a definition had been proposed in general terms by Deligne [8]. Theorem 2 specializes to give the following equivalence of categories.

Theorem 11. If the Beilinson-Soulé conjecture holds for $\operatorname{Spec}(F)$, then $\mathscr{M} \mathscr{T} \mathscr{M}(F)$ is equivalent to the category $\mathscr{F}_{\mathscr{H}}$.

Deligne [9] first suggested that, if a suitable commutative DGA $\mathscr{N}_{\mathbb{Q}}$ could in fact be constructed, then $\mathscr{F}_{\mathscr{H}} \mathscr{N}_{\mathbb{Q}}$ should give an appropriate definition of $\mathscr{M} \mathscr{T} \mathscr{M}(F)$ when the Beilinson-Soulé conjecture holds for $\operatorname{Spec}(F)$. Thus Theorem 11 is the promised equivalence of two approaches to mixed Tate motives. In view of (9), Corollary 4 has the following immediate consequence.

Theorem 12. If $\mathscr{N}_{\mathbb{Q}}$ is a $K(\pi, 1)$, then

$$
\operatorname{Ext}_{\mathscr{M} \mathscr{M}(F)}^{p}(\mathbb{Q}, \mathbb{Q}(r)) \cong g r_{\gamma}^{r}\left(K_{2 r-p}(F) \otimes \mathbb{Q}\right) .
$$

This verifies one of the key properties desired of a category of mixed Tate motives. Our Conjecture 5 suggests that there is a definition of mixed Tate motives that will lead to such an isomorphism without the $K(\pi, 1)$ hypothesis.

The results of [5] start from Definition 10 and give realization functors from $\mathscr{M} \mathscr{T} \mathscr{M}(F)$ to the category of mixed Tate $\ell$-adic representations in étale theory and to the category of mixed Tate Hodge structures in Hodge theory.

Étale realization. Fix a prime $\ell$ and assume that the characteristic of $F$ is prime to $\ell$ and that $F$ has finitely many $\ell$ th roots of unity. There is a rational Hopf algebra $\chi_{\text {ét }}$ such that the category of mixed Tate $\ell$-adic representations is equivalent to the category of finite-dimensional comodules 
over $\chi_{\text {ét }}$. Modulo replacing $\mathscr{N}_{\mathbb{Q}}$ by a quasi-isomorphic DGA, [5] constructs a suitable realization map of Hopf algebras

$$
\operatorname{Real}_{e ́ t}: \chi_{m o t} \rightarrow \chi_{e ́ t} .
$$

Hodge realization. Let $F=\mathbb{C}$. The category of mixed Tate Hodge structures is equivalent to the category of finite-dimensional comodules over a rational commutative Hopf algebra $\chi_{T M H}$. Again, modulo replacing $\mathscr{N}_{\mathbb{Q}}$ by a quasi-isomorphic DGA, [5] constructs a suitable realization map of Hopf algebras

$$
\operatorname{Real}_{T M H}: \chi_{m o t} \rightarrow \chi_{T M H}
$$

The desired realization functors on $\mathscr{M} \mathscr{T} \mathscr{M}(F)$ are obtained by passage to the associated categories of comodules or, equivalently, of generalized nilpotent representations of co-Lie algebras of indecomposable elements. We believe that stronger realization results are true. There are étale and Hodge analogues, $\mathscr{N}_{\mathbb{Q} \text {,ét }}$ and $\mathscr{N}_{\mathbb{Q}, T M H}$, of the DGA $\mathscr{N}_{\mathbb{Q}}$, and our Theorem 2 gives interpretations of mixed Tate $\ell$-adic representations and mixed Tate Hodge structures in terms of the derived categories of these DGA's. Although complete details are not in place, it appears that realization maps can be constructed on the level of DGA's, from which the displayed realization maps are obtained by application of the functor $H^{0} \bar{B}$. In fact, we believe that there is an integral version of this picture.

Conjecture 13. There are $E_{\infty}$ algebras $\mathscr{N}_{\text {ét }}$ and $\mathscr{N}_{T M H}$ together with quasi-isomorphisms of $E_{\infty}$ algebras

$$
\mathscr{N}_{\text {ét }} \otimes \mathbb{Q} \rightarrow \mathscr{N}_{\mathbb{Q}, e ́ t} \text { and } \mathscr{N}_{T M H} \otimes \mathbb{Q} \rightarrow \mathscr{N}_{\mathbb{Q}, T M H}
$$

There are also realization maps of $E_{\infty}$ algebras

$$
\text { Realét }_{\text {en }} \rightarrow \mathscr{N}_{\text {ét }} \text { and } \operatorname{Real}_{T M H}: \mathscr{N} \rightarrow \mathscr{N}_{T M H}
$$

that are compatible under rationalization with realization maps of DGA's

$$
\text { Realét }: \mathscr{N}_{\mathbb{Q}} \rightarrow \mathscr{N}_{\mathbb{Q}, e ́ t} \text { and } \operatorname{Real}_{T M H}: \mathscr{N}_{\mathbb{Q}} \rightarrow \mathscr{N}_{\mathbb{Q}, T M H}
$$

The reinterpretation of Definition 10 given by Theorem 11 leads to a category of integral mixed Tate motives that is related to $\mathscr{M} \mathscr{T} \mathscr{M}(F)$ by extension of scalars. In fact, we have constructed a derived category $\mathscr{D}_{A}$ associated to an $E_{\infty}$ algebra $\mathrm{A}$ over any commutative ring $k$. The relevant theory is precisely analogous to the topological theory of $E_{\infty}$ module spectra over an $E_{\infty}$ ring spectrum described in [12]. One first defines a 
category of " $E_{\infty} k$-modules". This is quite different from the category of $k$-modules, but it has an equivalent derived category: $k$-modules are the analogues of spectra and $E_{\infty} k$-modules are the analogues of $S$-modules. The category of $E_{\infty} k$-modules has its own tensor product, which is associative and commutative but only unital up to quasi-isomorphism. There is a tensor category of unital $E_{\infty} k$-modules, and an $E_{\infty}$ algebra $A$ is exactly a commutative monoid in this category. From here, the treatment of $A$-modules proceeds in terms of the new $E_{\infty}$ tensor product over $k$. Just as if $A$ were an actual DGA, the derived category $\mathscr{D}_{A}$ is a triangulated tensor category.

Deligne $([9],[10, \S 3])$ proposed the resulting category $\mathscr{D}_{\mathscr{N}}$ as an integral "catégorie triangulée motivique". Given our Conjecture 13, this definition will lead directly to étale and Hodge realization functors. We define Adams graded Ext groups in terms of the morphisms of this category:

$$
\operatorname{Ext}_{\mathscr{N}}^{q}(M, N)=\mathscr{D}_{\mathscr{N}}(M, N[q])
$$

for $\mathscr{N}$-modules $M$ and $N$. These agree with the cohomology groups of the right derived $\mathscr{N}$-module $\operatorname{Hom}_{\mathscr{N}}(M, N)$, and we have a spectral sequence that converges from

$$
\operatorname{Ext}_{H^{*}(\mathscr{H})}^{*, *}\left(H^{*}(M), H^{*}(N)\right)
$$

to $\operatorname{Ext}_{\mathscr{N}}^{*}(M, N)$. Here $H^{*}(\mathscr{A})$ is the integral Chow ring of $\operatorname{Spec}(F)$, regraded as dictated by formulas (8) and (9). Little is known about the integral Chow groups, and there seems to be only speculation as to their relationship to the higher algebraic $K$-groups of $F$. However, the results on derived categories just outlined work equally well if we reduce $\bmod n$, and Suslin [16] has recently proven that if $F$ is an algebraically closed field of characteristic prime to $n$ and $X$ is a smooth affine variety over $F$, then, for $r \geq \operatorname{dim}(X)$,

$$
C H^{r}(X, q ; \mathbb{Z} / n) \cong H_{e ́ t}^{2 r-q}(X, \mathbb{Z} / n(r)) .
$$

Working topologically [12], we have constructed an analogous "derived category" that bears the same relation to the $K$-groups $K_{*}(F)$ that $\mathscr{D}_{\mathscr{N}}$ bears to the Chow groups. This is the stable homotopy category $\bar{h} K(F)$ of module spectra over the $E_{\infty}$ ring spectrum $K(F)$ that represents the algebraic K-theory of $F$. It provides another plausible candidate for a motivic triangulated tensor category. We have a function $E_{\infty}$ module spectrum $F_{K(F)}(M, N)$ for $E_{\infty}$ module spectra $M$ and $N$. Its homotopy groups are the "topological Ext groups"

$$
\operatorname{Ext}_{K(F)}^{q}(M, N) \equiv\left[M, \Sigma^{q} N\right]_{K(F)},
$$


and we have a spectral sequence that converges from

$$
\operatorname{Ext}_{K_{*}(F)}^{*, *}\left(M_{*}, N^{*}\right)
$$

to $\operatorname{Ext}_{K(F)}^{*}(M, N)$. Despite the tantalizingly close analogy, we do not yet see how to forge a connection between the candidates $\mathscr{D}_{\mathscr{N}}$ and $\bar{h} K(F)$ for categories of integral mixed Tate modules.

\section{Acknowledgement}

It is a pleasure to thank Deligne for letters that led to this work and to thank Bloch for numerous discussions of it.

\section{References}

1. A. A. Beilinson, J. Bernstein, and P. Deligne, Faisceaux pervers, Astérisque 100 (1982), 5-171.

2. J. Bernstein and V. Lunts, Equivariant sheaves and functors, preprint, 1992.

3. S. Bloch, Algebraic cycles and higher K-theory, Adv. Math. 61 (1986), 267-304.

4. _ The moving lemma for higher Chow groups, preprint, 1993.

5. S. Bloch and I. Kriz, Mixed Tate motives, Annals of Math. (to appear).

6. A. Borel, Stable real cohomology of arithmetic groups, Ann. Sci. École Norm. Sup. 7 (1974), 235-272.

7. P. Deligne, Catégories tannakiennes, The Grothendieck Festschrift, vol. II, Birkhauser, 1990, pp. 111-195.

8. __ Letter to Beilinson dated December 6, 1989.

9. __ Letters to Bloch and May dated March 19, March 20, April 25, and May 2, 1990.

10. _ A quoi servent les motifs?, Proceedings of the Summer, 1991, conference on motives, University of Washington (to appear).

11. P. Deligne and J. S. Milne, Tannakian categories, Springer Lecture Notes in Mathematics, vol. 900, 1982, pp. 101-228.

12. A. Elmendorf, J. P. C. Greenlees, I. Kriz, and J. P. May, Commutative algebra in stable homotopy theory and a completion theorem, Mathematical Research Letters (to appear).

13. M. Levine, Bloch's higher Chow groups revisited, preprint, 1993.

14. J. P. May (with contributions by F. Quinn, N. Ray, and J. Törnehave), $E_{\infty}$ ring spaces and $E_{\infty}$ ring spectra, Springer Lecture Notes, vol. 577, 1977.

15. D. Sullivan, Infinitesimal computations in topology, Publ. Math. IHES 47 (1978), 269-331.

16. A. Suslin, Higher Chow groups of affine varieties and étale cohomology, preprint, 1993.

University of Chicago, Chicago, IL 60637

E-mail address: kriz@math.uchicago.edu and may@math.uchicago.edu 